\title{
Substituent effects on the oxidation of substituted biphenyl congeners by type II methanotroph strain CSC1
}

Received: 27 October 2004/ Revised: 22 February 2005/ Accepted: 4 March 2005/Published online: 15 April 2005

(C) Springer-Verlag 2005

\begin{abstract}
The oxidation potential of type II groundwater methanotroph, strain CSC1, expressing soluble methane monooxygenase, was measured in the presence of 10 ortho-substituted biphenyls with varying electronics, sterics, and hydrophobicity character for comparison with type II Methylosinus trichosporium OB3b. Strain $\mathrm{CSC} 1$ showed faster rates with all compounds tested, with the exception of 2-nitrobiphenyl, 2-hydroxybiphenyl, and 2-aminobiphenyl. Products of oxidation observed upon incubation of strain CSC1 with biphenyl and 2-hydroxybiphenyl were hydroxylated biphenyls that revealed less preference for the para position and different dihydroxylation positions, respectively, in comparison to those observed with $M$. trichosporium OB3b. Only the intramolecular hydrogen migration, or NIH-shift, product was observed in the case of 2-chlorobiphenyl, whereas $M$. trichosporium OB3b yielded a variety of chlorohydroxybiphenyls. Quantitative structure-biodegradation relationships constructed with the maximum observed oxygen uptake rates as a dependent variable and a variety of descriptors showed an influence of substituent electronic character on the oxidation activity of strain CSC1. However, compound hydrophobicity and not compound size, as was observed with $M$. trichosporium OB3b, was shown to influence rates to a greater extent. This suggests that transport of the compound through the cell membrane and to the sMMO active site is rate-determining for strain CSC1.
\end{abstract}

\footnotetext{
A. S. Lindner $(\bowtie)$

Department of Environmental Engineering Sciences, University of Florida, A.P. Black Hall,

P.O. Box 116450, Gainesville,

FL 32611-6450, USA

E-mail: alind@eng.ufl.edu

Tel.: + 1-352-8463033

Fax: +1-352-3923076

J. D. Semrau · P. Adriaens

Department of Civil and Environmental Engineering, University of Michigan, 1351 Beal Ave., Suite 181, Ann Arbor, MI 48109-2125, USA
}

Keywords Biodegradation - Methanotrophic oxidation - Molecular orbital descriptors Multivariate analysis - Quantitative structurebiodegradation relationships $\cdot$ Soluble methane monooxygenase

\section{Introduction}

Methane-oxidizing bacteria, or methanotrophs, thrive in environments where there are stable sources of oxygen and methane, their sole source of carbon and energy for growth. These bacteria have been termed "ubiquitous," and, as a result, have been implicated in degrading a significant quantity of methane before its release into the atmosphere (Conrad 1996; Reeburgh 1996). The role of methanotrophs in the environment has also been extended to cometabolic degradation of other contaminant classes, including aliphatic and aromatic compounds (Burrows et al. 1984; Green and Dalton 1989).

Methanotrophs have been classified into two broad categories, types I or II, depending on many factors, including metabolic pathways, cell morphology, and 16S rDNA sequences (Bowman et al. 1993; Bowman 2000). The enzyme responsible for the first step in methane oxidation is methane monooxygenase (MMO). Almost all known methanotrophs possess a particulate membrane-associated form of MMO (pMMO), whereas some methanotrophs can also express a soluble form of MMO (sMMO), under low concentrations of copper (Stanley et al. 1983; Prior and Dalton 1985; Choi et al. 2003). Type II methanotrophs expressing sMMO have been shown to cometabolically oxidize both aliphatic and aromatic compounds and, thus, show promise in treatment processes involving bioremediation of a wide range of contaminant classes (Green and Dalton 1989; DiSpirito et al. 1992; Bowman and Sayler 1994; Brigmon 2002).

Despite the commonality of expression of sMMO under low copper concentrations among methanotrophs 
capable of expressing this enzyme, the functional range of substrate oxidation diverges. For example, whole cells of Methylocystis strain M expressing sMMO were found to be incapable of oxidizing the aromatic compounds benzene, chlorobenzene, phenol, and para-chlorobiphenyl (Uchiyama et al. 1989), whereas sMMO isolated from Methylocystis sp. strain WI 14 has been shown to oxidize a variety of benzene and naphthalene compounds (Grosse et al. 1999). Whole cells of Methylosinus trichosporium $\mathrm{OB} 3 \mathrm{~b}$ expressing sMMO have been reported to cometabolize various aromatics, including biphenyl compounds (Dalton et al. 1993; Lindner et al. 2000). Whereas previous work has identified various pathways of oxidation followed by sMMO in whole cells of some type II methanotrophs (Dalton et al. 1981; Jezequel and Higgins 1983; Adriaens 1994; Wilkins et al. 1994), scant attention has been paid to linking variation in oxidation activities and products among these methanotrophs expressing sMMO to physiological differences among the strains.

Recent studies have reported the success of quantitative structure-biodegradation relationships (QSBRs) in providing a comparative platform to explain differences in activities among bacteria (Hermens et al. 1995; Damborsky et al. 1996). The QSBRs, correlations that relate rates to the electronic, steric, and hydrophobicity character of compounds composing a training set, enable determination of the rate-determining step - e.g., uptake by and transport within the cell and bonding to and/or transformation at the active site - involved in the degradation of substrates by whole cells (Hermens et al. 1995). In turn, a comparison of the rate-limiting step provides insight into differences in cell physiology and enzyme active-site structure and mechanism among bacteria while also potentially providing predictive capabilities for other compounds not in the training set. The hypothesis of this study was that QSBRs might provide an increased understanding of the physiological and/or mechanistic causes of the differences in aromatic substrate ranges observed among selected type II methanotrophs.

The specific focus of this work was a groundwater methanotroph, strain CSC1, originally isolated from an uncontaminated aquifer and subsequently shown to be capable of oxidizing aromatic compounds, including chlorobiphenyls and linear alkylbenzene sulfonates (Henry and Grbić-Galić 1990; Adriaens and Grbić-Galić 1994; Hršak 1996). The objectives of this work were to measure the rates and products of oxidation by strain CSC1 with a variety of ortho-substituted biphenyls, to quantify the influence of electronics, sterics, and hydrophobic character of the substrate on oxidation rates using QSBR multivariate analysis, and to compare these relative influences to those previously determined for $M$. trichosporium $\mathrm{OB} 3 \mathrm{~b}$, thus providing a first basis for the comparison and ultimate prediction of methanotroph activity under sMMO expression. Here, we show differences in substituent influences on the oxidation activities of strain CSC1 and M. trichosporium OB3b and discuss the possible significance of our findings in terms of known differences in whole-cell physiology of the two strains.

\section{Materials and methods}

\section{Culturing of strain CSC1}

Strain CSC1 was grown in nitrate mineral salts (NMS) medium with no added copper at $30^{\circ} \mathrm{C}$, as described previously (Lindner et al. 2000). To verify that sMMO was responsible for the oxidation of the aromatic substrates, strain CSC1 was also cultured in the presence of $20 \mu \mathrm{M}$ copper as $\mathrm{Cu}\left(\mathrm{NO}_{3}\right)_{2}$ to allow expression of pMMO. The naphthalene assay specific for sMMO activity was used to monitor whole-cell expression of sMMO in all cell suspensions (Brusseau et al. 1990). For both oxygen uptake and transformation studies, cells were collected by centrifugation $(6,000 \mathrm{~g}$ for $30 \mathrm{~min})$ and resuspended to $0.2 \mathrm{~g}$ (wet wt) $\mathrm{ml}^{1}$ in phosphate buffer.

\section{Chemicals}

\section{Substituted biphenyl compounds}

As shown in Table 1, the training set of compounds chosen for study represents a series of biphenyls with ortho substituents that vary broadly in electronics, sterics, and hydrophobicity. These compounds include substituents that span wide ranges of inductive effects (from 2-fluorobiphenyl and 2-nitrobiphenyl to 2-aminobiphenyl), hydrophobicity (from 2-nitrobiphenyl to 2iodobiphenyl and 2-methylbiphenyl), and size (from 2fluorobiphenyl to 2-iodobiphenyl). Biphenyl was chosen as an unsubstituted reference compound.

In addition, biphenyl, 2-chlorobiphenyl, and 2-hydroxybiphenyl were selected for resting-cell transformation studies with strain CSC1. The primary objective of these experiments was to compare product type and relative abundance to that previously observed with M. trichosporium OB3b (Lindner et al. 2000). Biphenyl was chosen for product analysis to compare preference of the 2 position, 3 position, or 4 position in oxidation. 2-Chlorobiphenyl was chosen to assess the relative abundance of the intramolecular hydrogen migration, or NIH-shifted product, previously reported by Adriaens (1994), in relation to other hydroxylated products, and 2-hydroxybiphenyl was selected to assess the ability of strain CSC1 to form dihydroxylated biphenyls.

\section{Other chemicals}

All chemicals used in media preparation were of reagent grade or better quality. Highest purity methane (at least 99.99\%) was obtained from Matheson Gas Products (Montgomeryville, Pa., USA). Distilled-deionized water 
Table 1 Resonance parameter $(R)$, atomic charges $\left(C_{i}\right)$, molar refractivity $(M R)$, and $\log K_{\mathrm{ow}}$ of compounds in training set

\begin{tabular}{|c|c|c|c|c|c|c|c|c|c|}
\hline Compound $^{\mathrm{a}}$ & $R^{\mathrm{f}}$ & $\mathrm{C}_{8}^{\mathrm{b}, \mathrm{c}}$ & $\mathrm{C}_{9}$ & $\mathrm{C}_{10}$ & $\mathrm{C}_{11}$ & $\mathrm{C}_{12}$ & $\delta_{C-\mathrm{X}}^{\mathrm{d}}$ & $\mathrm{MR}^{\mathrm{f}}$ & $\log K_{\text {ow }}^{\mathrm{f}}$ \\
\hline BP & 0.00 & -0.0640 & -0.0633 & -0.0632 & -0.0633 & -0.0640 & -0.1266 & 0.10 & 4.01 \\
\hline $2 \mathrm{FBP}$ & -0.39 & -0.0565 & -0.0702 & -0.0550 & -0.0870 & 0.1385 & 0.2729 & 0.09 & 4.00 \\
\hline $2 \mathrm{CBP}$ & -0.19 & -0.0584 & -0.0586 & -0.0583 & -0.0533 & 0.0457 & 0.2093 & 0.60 & 4053 \\
\hline 2BBP & -0.22 & -0.0598 & -0.0626 & -0.0604 & -0.0598 & -0.0467 & -0.0430 & 0.89 & 4.59 \\
\hline $2 \mathrm{IBP}$ & -0.24 & -0.0605 & -0.0622 & -0.0614 & -0.0592 & -0.0877 & -0.1327 & 1039 & 4.98 \\
\hline 2NBP & 0.13 & -0.0574 & -0.0522 & -0.0562 & -0.0520 & 0.835 & -0.0421 & 0.74 & 2.54 \\
\hline 2MBP & -0.18 & -0.0631 & -0.0680 & -0.0638 & -0.0678 & 0.01333 & -0.1719 & 0.56 & 4.52 \\
\hline 2HBP & -0.70 & -0.0548 & -0.0764 & -0.0529 & -0.0996 & 0.12353 & 0.4022 & 0.28 & 3.23 \\
\hline $2 \mathrm{ABP}$ & -0.74 & -0.0638 & -0.0651 & -0.0635 & -0.0788 & 0.08004 & 0.4664 & 0.54 & 2.84 \\
\hline $2 \mathrm{MxBP}$ & -0.56 & -0.0569 & -0.0763 & -0.0540 & -0.0998 & 0.12630 & 0.3659 & 0.79 & 3.91 \\
\hline
\end{tabular}

${ }^{a} B P$ biphenyl, $2 F B P$ 2-fluorobiphenyl, 2CBP 2-chlorobiphenyl, 2BBP 2-bromobiphenyl, 2IBP 2-iodobiphenyl, 2NBP 2-nitrobiphenyl, $2 M B P$ 2-methylbiphenyl, $2 H B P$ 2-hydroxybiphenyl, $2 A B P$ 2-aminobiphenyl, 2-MxBP 2-methoxybiphenyl

${ }^{\mathrm{b}}$ Carbon numbers on substituted ring of biphenyl $\left(C_{i}\right)$

${ }^{\mathrm{c}}$ Calculated using Gaussian 94 software (Gaussian 1996). Units of $C_{i}$ in eV

${ }^{\mathrm{d}}$ The difference between the substituted carbon charge $\left(C_{12}\right)$ and the charge of the substituent atom bonded with $\mathrm{C}_{12} . X$ refers to the ortho substituent

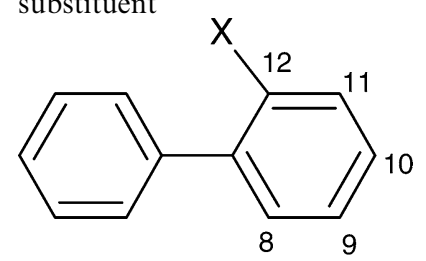

${ }^{\mathrm{f}}$ Hansch et al. (1995)

from a Corning Millipore D2 system (Billerica, Mass., USA) was used for all experiments. The biphenyl substrates were purchased from AccuStandard (New Haven, Conn., USA), ICN Biochemicals (Aurora, Ohio, USA), Pfaltz \& Bauer (Waterbury, Conn., USA), Sigma-Aldrich (St. Louis, Mo., USA), and UltraScientific (N. Kingstown, R.I., USA).

\section{Measurement of oxygen uptake rates}

A custom designed 1.9-ml glass, water-jacketed reactor, equipped with a Clarke-type electrode (Instech Laboratories, Plymouth, Mass., USA) and an automated data collection system, were used at a constant temperature of $30^{\circ} \mathrm{C}$ to measure the rates of oxygen consumption at various initial substrate concentrations. A two-point calibration of the electrode was performed daily and after application of fresh electrolyte and membrane by equilibration with the atmosphere $(100 \%)$ and dosing with saturated sodium sulfite solution $(0 \%)$.

To further verify that sMMO was responsible for oxidation of biphenyl, cells expressing sMMO were inactivated with acetylene as described previously (Lontoh et al. 1999), and oxygen uptake was measured in the presence of substrate. As an additional control, strain $\mathrm{CSC} 1$ grown in the presence of $20 \mu \mathrm{M}$ copper was added to the oxygen uptake reactor at a concentration of $0.2 \mathrm{~g}$ (wet wt) $\mathrm{ml}^{1}$ and mixed with substrate. Verification of observed oxygen uptake by sMMO was determined by no observed activity in the presence of acetylene-treated and copper-grown cells.
Oxygen uptake in the capped reactor was monitored in the presence of methane or the aromatic compounds. Methane was added by bubbling $4 \mathrm{ml}$ of methane into the cell suspensions to obtain $1.4 \mathrm{mM}$ methane in solution. The substrate was added in a solution with 1,4-dioxane solvent (shown to cause no probe effect or to be oxidized by strain CSC1) in different volumes to yield a range of concentrations, and the initial rates of oxygen uptake were measured. During the assays, it was noticed that microbial activity decreased by up to $50 \%$ over $8 \mathrm{~h}$, as measured by a decrease in oxygen uptake in the presence of $1.4 \mathrm{mM}$ methane, possibly because of the decay of active sMMO after cell harvesting. As described in more detail previously (Lindner et al. 2000), to account for this variation when comparing oxygen uptake in the presence of different substrates, the rates shown here are normalized to the rate of oxygen uptake in the presence of $1.4 \mathrm{mM}$ methane, measured just prior to the measurement of oxygen uptake upon change to an increased concentration of substrate. To prevent additional loss of cell activity, the resuspended cells were kept on ice throughout the oxygen uptake experiments.

The oxygen uptake rates were determined by calculating the linear slope of the initial data points over a time period of 60-300 s, depending on the substrate, and this initial rate was, in turn, corrected for endogenous metabolism, i.e., the amount of oxygen used by strain CSC1 before the addition of any substrate. All rates of oxygen uptake exhibited by $M$. trichosporium $\mathrm{OB} 3 \mathrm{~b}$ with the same training set of ortho-substituted biphenyls were 
previously measured and reported by Lindner et al. (2003). These rates were used in this study for correlation analysis.

\section{Resting-cell transformation studies}

Resting-cell incubations of strain CSC1 with biphenyl, 2-chlorobiphenyl, and 2-hydroxybiphenyl were conducted in sterile, acid-rinsed 160 -ml serum vials in order to identify products and their relative distribution. Resuspended cells $(100 \mu \mathrm{l})$ were removed to measure oxygen uptake activity in the presence of methane as previously described. The remaining resuspended cells were diluted with NMS medium to a concentration of 1.4-2.3 mg (wet wt) $\mathrm{ml}^{1}$. The vials were filled with 20 $30 \mathrm{ml}$ of this dilute cell suspension and supplemented with different substrates at the concentration where maximum oxygen uptake was observed. Three vials were sealed with Teflon-lined red rubber septa and incubated at $30^{\circ} \mathrm{C}$ and $270 \mathrm{rpm}$ for 2 days. Another vial, serving as a time zero sample, was quenched with 20 $30 \mathrm{ml}$ of hexane and immediately frozen. Further, a killed control was prepared using autoclaved cells, and a chemical control was prepared using NMS medium and substrate.

After sample incubation, a 5-ml aliquot was removed from each microcosm and subjected to three hexane extractions $(1: 1, \mathrm{v} / \mathrm{v})$ to measure the amount of substrate degraded. These extracts were pooled, dried over anhydrous $\mathrm{MgSO}_{4}$, and concentrated to $0.1 \mathrm{ml}$ using a rotoevaporator and a thermoevaporator (heated at $60^{\circ} \mathrm{C}$ ) with a gentle stream of $\mathrm{N}_{2}$. To determine oxidative product(s), the remainder of each of the incubation mixtures was prepared by acidification to $\mathrm{pH} 2$, "salting out" with a saturated $\mathrm{NaCl}$ solution, extraction with ethyl acetate (Fisher Scientific, Pittsburgh, Pa., USA), and subsequent evaporation. This sample preparation method is described in more detail in Lindner et al. (2000). If products were difficult to detect in these extracts, they were dried using thermoevaporation at $60^{\circ} \mathrm{C}$ and then derivatized by the addition of $N$-trimethylsilylimidazone [(TMSI), Alltech, Deerfield, Ill., USA] and acetonitrile, with subsequent heating at $60^{\circ} \mathrm{C}$ (Kohler et al. 1988, 1993; Kitson et al. 1996).

Prepared samples were then analyzed on a mass spectrometer (Model MSD, Hewlett Packard, Palo Alto, Calif., USA), operated at $70 \mathrm{eV}$, and interfaced to a gas chromatograph (model 5890, Hewlett Packard) with a 30 m DB-5 capillary column (J \& W Scientific, Folsom, Calif., USA) as described earlier (Lindner et al. 2000). When standards were not available, the product(s) of transformation were identified using a combination of linear $(100-500 \mathrm{~m} / \mathrm{z})$ and selective ion-monitoring (SIM) analysis. During SIM analysis, masses in both the molecular ion cluster and the major fragments (loss of halogen, loss of TMSI, etc.) were used as fingerprints for the potential product(s).
Correlation analysis for determination of quantitative structure-biodegradation relationships

\section{Atomic and molecular descriptors}

Electronic effects, size, and hydrophobicity of the compounds were represented in the correlations because of their expected significance in the mechanism of substrate transformation by whole cells, considering the steps involving transport across the cell membrane and to the enzyme active site and binding, reaction, and release at the active site (Damborsky et al. 1996). Those empirical descriptors of electronic effects, size, or hydrophobicity with more than one value provided in source references were chosen based on common measurement methods and experimental conditions.

Electronic descriptors that were used accounted for both mesomeric and inductive effects of the substituent, potential factors impacting the rate of enzymatic conversion of the compound. Unless otherwise noted, these descriptors were obtained from Hansch et al. (1995). Empirical parameters measuring resonance effects were represented by Hammett constants, $\sigma_{\mathrm{o}}, \sigma_{\mathrm{m}}, \sigma_{\mathrm{p}}$, and $\Sigma \sigma$, the sum of $\sigma_{\mathrm{m}}$ and $\sigma_{\mathrm{p}}$. The resonance parameter $R$ was also used to account for mesomeric effects of the substituent (Table 1). The Hammett constant $\sigma_{\mathrm{I}}$ and the field parameter $F$ were used to describe inductive effects of the substituent on the aromatic ring, which would be expected in the case of strongly electron-donating and electron-withdrawing substituents.

Electronic effects of the substituent were also represented by quantum mechanical descriptors derived for this study using molecular orbital calculations. The charge on the aromatic carbon atoms (Table 1) and dipole moment $\mu$ were calculated using Gaussian 94 software (Gaussian, Pittsburgh, Pa., USA, Gaussian 1996). After selection of the Hamiltonian operator, basis set, and molecular system to be studied, calculations were applied on all molecules of the training set with a basis set of $\mathrm{HF} / 3-21 \mathrm{G}(\mathrm{d}) / \mathrm{HF} / 3-21 \mathrm{G}(\mathrm{d}, \mathrm{p})$. Each computation began with a single-point calculation, followed by geometric optimization and calculation of the atomic and molecular properties to be used as descriptors in the QSBRs. Procedural details of calculations can be reviewed in the Gaussian 94 manual that accompanies the software (Foresman and Frisch 1996). As shown in Table 1, a specific numbering system was chosen for the carbons on the substituted ring, ranging from $\mathrm{C}_{8}$, denoting the non-substituted ortho carbon, to $\mathrm{C}_{12}$, denoting the substituted ortho carbon. Thus, the sum of all benzene carbon charges on the substituted ring was denoted by $\mathrm{SUMC}_{8}: \mathrm{C}_{12}$, and the sum of charges of benzene carbons at the substituted site and ortho, meta, and para to this site were denoted by $\mathrm{SUMC}_{9}: \mathrm{C}_{12}$. The difference in charges on the substituted carbon and the substituent $\left(\delta_{C-\mathrm{X}}\right)$ was also included to assess the relative importance of this position during oxidation by the whole cells. Correlations were constructed using both individual carbon charges as well as these sums of car- 
bon charges as a means of assessing the impact of electron density on the activity of strain CSC1 whole cells with the test compounds.

Steric descriptors used to account for rate-limiting effects in passage through the cell membrane as well as in the enzyme active site included the Taft steric constant and the molar refractivity of the substituent obtained from Hansch et al. (1995). Molecular orbital-derived parameters were also used to describe substituent size were the ortho-carbon-substituent bond length $(\mathrm{C}-\mathrm{X})$ and width of compound. The latter was determined by the dihedral angles and bond lengths provided by the molecular orbital calculations (Table 1). Descriptors representing hydrophobicity of the compound included $\log K_{\text {ow }}$ (Table 1) and the constant $\pi$ (Hansch et al. 1995).

\section{Correlation development and validation}

Upon establishing the substrate-descriptor matrices, a rigorous screening of descriptors was performed using linear and multivariate regression methods. SPSS software (SPSS, Chicago, Ill., USA) was used to construct the correlations with the oxygen uptake rates by strain CSC1 in the presence of each substrate serving as the activity parameter (dependent variable). $\log \left(k_{x} / k_{H}\right)$ was always used as the dependent variable, where $k_{\mathrm{x}}$ and $k_{H}$ are the resulting activity parameters in the presence of the substituted biphenyl and biphenyl, respectively. Both the slope fitted to the initial rates (including the maximum observed rate) and the maximum observed rates were tested for their suitability as a dependent variable. Each training set was composed of the nine compounds (see Table 1) with biphenyl as the parent compound. By using all possible combinations of electronic, steric, and hydrophobicity descriptors, numerous QSBRs were constructed for each data set.

Three sets of statistical criteria were used to reduce the strain CSC1 QSBR matrix and to determine the "first-cut" correlations: (1) $r^{2}>0.70$; (2) $F$-ratio (calculated) $>F_{\alpha n, n-k-1}$ [where $\alpha$ is the confidence imposed on the correlation (no more than 0.1 for this study), $k$ the degrees of freedom for the regression, $n$ the number of compounds tested, and $n-k-1$ is the degrees of freedom for the residual]; and (3) the collinearity of any two independent variables in a correlation must be low (as represented by $r^{2}<0.4$ ). This smaller list of correlations was then subjected to more stringent criteria based on the highest $F$-ratios, lowest $95 \%$ confidence interval on coefficients, and low covariance of the descriptors. In addition, using the training set of nine compounds, only correlations with one or two independent variables were deemed suitable for the final set of acceptable models, as described by Topliss and Edwards (1979) and Hermens et al. (1995). The final QSBRs were then tested for outliers by comparing $r^{2}$ values, mean square residuals, and the standard errors of the coefficients of the original correlation and new correlations resulting from removing any suspect data point (Montgomery and Peck 1982; Taylor 1990). The correlations were also subjected to cross-validation by calculation of $Q^{2}$ values as a means of assessing predictive capability (Montgomery and Peck 1982; Taylor 1990; Eriksson et al. 1997, 2003). It is important to note that the "leave-one-out" method was applied in this study for cross-validation. Whereas recognized as often yielding $Q^{2}$ values similar to $r^{2}$ values, the number of compounds in the data set for this study $(n=9)$ is too small for the recommended "leave-moreout" approach (Eriksson et al. 2003). Models were deemed of good predictive value (and not by chance) if the $Q^{2}$ value were greater than 0.5 and the difference between $r^{2}$ and $Q^{2}$ values were no greater than 0.3 (Eriksson et al. 2003). All correlations passing these criteria were plotted to show deviations from the model and the data (residuals).

\section{Results and discussion}

Substituent effects on oxidation kinetics

Figure 1 shows the normalized rates of oxygen uptake by whole cells of strain $\mathrm{CSC1}$ in the presence of a range of biphenyl, 2-chlorobiphenyl, and 2-hydroxybiphenyl concentrations. As previously described, these substrates were chosen for both rate and product analysis. The ratesubstrate concentration curves obtained for all nine biphenyl substrates exhibited a clear maximum observed rate, as shown in Fig. 1 for biphenyl, 2-chlorobiphenyl, and 2-hydroxybiphenyl, with depressed rates at higher

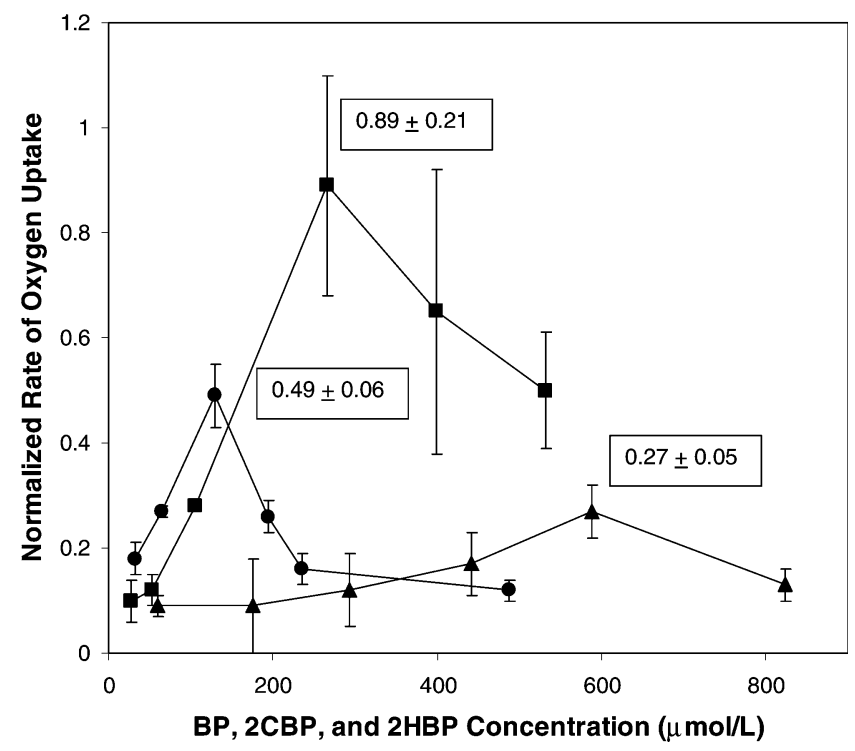

Fig. 1 Oxygen uptake rates of strain CSC1 in the presence of biphenyl $[(B P)$ filled circles], 2-chlorobiphenyl [(2CBP) filled squares], and 2-hydroxybiphenyl [(2HBP) filled triangles]. Maximum rates are shown in the boxes. Error bars represent the standard deviation of a minimum of triplicate samples 
substrate concentrations. This behavior does suggest a toxicity effect of these substrates, possibly rendered by the substrate itself or by toxic intermediates formed. Because rates were calculated using initial data points collected as previously described, formation of intermediates was not anticipated to play a major role in toxicity effects. However, the role of substrate toxicity is not clear, because the rates were not shown to fit substrate toxicity models, as was also observed previously with M. trichosporium OB3b (Lindner et al. 2000).

The maximum rates of oxygen uptake by strain CSC1 observed in the presence of the biphenyl compounds normalized to oxygen uptake rates in the presence of methane ranged from $0.09+0.02$ with 2-nitrobiphenyl to $1.25 \pm 0.15$ with 2 -iodobiphenyl, and the concentration of the observed maxima ranged from $25 \mu \mathrm{M}$ with 2-nitrobiphenyl to $588 \mu \mathrm{M}$ with 2-hydroxybiphenyl (Table 2). Both biphenyls with substituents with greatest electron-withdrawing capacity $\left(-\mathrm{NO}_{2}\right.$ and $\left.-\mathrm{F}\right)$ yielded the lowest maximum oxygen uptake rates and respective substrate concentrations. These data show no clear trend that strain CSC1 prefers biphenyl compounds with substituents of certain electronic, steric, or hydrophobic character; however, an interesting trend is shown with the increase in rates as the ortho substituent progresses down the halogen series. This result is reminiscent of previous reports of the behavior of $M$. trichosporium OB3b (Lindner et al. 2000) that was also shown to favor 2-iodobiphenyl, completely dehalogenating this compound to yield only hydroxylated biphenyls.

The strain CSC1 rates in Table 2 diverge significantly from those previously observed with $M$. trichosporium OB3b; however, strain CSC1 displayed higher maximum oxygen uptake rates with all of the halogenated biphenyls than $M$. trichosporium OB3b, nearly four times faster with 2-chlorobiphenyl and approximately 1.5 times for the other halogenated biphenyls. M. trichos-

Table 2 Normalized oxygen uptake rates observed in the presence of each biphenyl substrate

\begin{tabular}{lll}
\hline Substrate & $\begin{array}{l}\text { Normalized maximum } \\
\text { oxygen uptake rate }\end{array}$ & $\begin{array}{l}\text { Substrate concentration } \\
\text { at maximum oxygen } \\
\text { uptake rate }(\mu \mathrm{M})\end{array}$ \\
\hline BP & $0.49 \pm 0.06$ & 130 \\
2FBP & $0.23 \pm 0.08$ & 43 \\
2CBP & $0.89 \pm 0.21$ & 266 \\
2BBP & $0.92 \pm 0.15$ & 515 \\
2IBP & $1.25 \pm 0.15$ & 268 \\
2NBP & $0.09 \pm 0.02$ & 25 \\
2MBP & $0.48 \pm 0.09$ & 595 \\
2HBP & $0.27 \pm 0.05$ & 588 \\
2ABP & $0.32 \pm 0.14$ & 444 \\
2MxBP & $0.35 \pm 0.05$ & 305
\end{tabular}

${ }^{a}$ Each rate represents the maximum observed oxygen uptake rate in the presence of substrate divided by the oxygen uptake rate observed in the presence of $1.4 \mathrm{mM}$ methane. The average oxygen uptake rate observed with $1.4 \mathrm{mM}$ methane was $0.03+0.01 \mu \mathrm{mo}-$ $1 \mathrm{O}_{2} / \mathrm{min}$. The error represents the standard deviation of a minimum of three data points porium OB3b showed higher oxidation potentials only with 2-nitrobiphenyl $(0.20+0.07)$, 2-hydroxybiphenyl $(0.61+0.001)$, and 2-aminobiphenyl $(2.14+0.43)$ (Lindner et al. 2003). Further differentiation of the rates observed with the two strains was subsequently possible using QSBR analysis as described to follow.

\section{Substituent effects on oxidation products}

Resting-cell transformations of biphenyl, 2-hydroxybiphenyl, and 2-chlorobiphenyl by strain CSC1 yielded a variety of substituted biphenyl products and allowed further comparison with $M$. trichosporium OB3b. As shown in Table 3, ortho- (2-position), meta(2-position), and para- (4-position) hydroxylation of biphenyl was observed, with para-hydroxybiphenyl in greatest abundance $(59 \%)$ compared to the abundances of 2-hydroxybiphenyl and 3-hydroxybiphenyl (29 and $12 \%$, respectively). These results reflect a ratio of $1.4: 1$ of products in favor of para-hydroxylation by strain CSC1, a far lower ratio than the 9:1 ratio observed with M. trichosporium OB3b (Lindner et al. 2000). The greater preference of the sMMO of $M$. trichosporium OB3b for the para position suggests a steric hindrance effect in the enzyme active site, where the ortho position and meta position are not as accessible.

Evidence of the ability of strain CSC1 to hydroxylate a hydroxylated aromatic compound was shown with incubation with 2-hydroxybiphenyl, where three dihydroxylated biphenyl isomers were observed in abundances of 49,34 , and $17 \%$, respectively (Table 3 ). These isomers could not be identified because of the lack of authentic standards for all possible dihydroxylated isomers; however, it was possible to conclude that none of these isomers were 3,4-dihydroxybiphenyl, 2,5-dihydroxybiphenyl, or 2,2'-dihydroxybiphenyl (for which standards were commercially available). None of the retention times and fragment intensities of the dihydroxylated products observed in strain CSC1 incubations compared to the products observed in $M$. trichosporium OB3b incubations, thus implying different isomers and hydroxylation patterns.

Table 3 Products of substituted biphenyl oxidation by strain CSC1

\begin{tabular}{|c|c|c|}
\hline Substrate $^{\mathrm{a}}$ & Products & $\begin{array}{l}\text { Relative } \\
\text { abundance }^{\mathrm{c}}(\%)\end{array}$ \\
\hline Biphenyl & $\begin{array}{l}\text { 2-Hydroxybiphenyl } \\
\text { 3-Hydroxybiphenyl } \\
\text { 4-Hydroxybiphenyl }\end{array}$ & $\begin{array}{l}29 \\
12 \\
59\end{array}$ \\
\hline 2-Hydroxybiphenyl & $\begin{array}{l}\text { Dihydroxylated } \\
\text { biphenyls (3) }\end{array}$ & 100 \\
\hline 2-Chlorobiphenyl & 2-Hydroxy-3-chlorobiphenyl & 100 \\
\hline
\end{tabular}

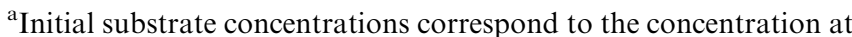
which the observed oxygen uptake rate was maximum

${ }^{\mathrm{b}}$ Derivatized samples

${ }^{\mathrm{c}}$ The percent of relative abundance values represent the distribution of observed products only 
2-Chlorobiphenyl incubation with strain CSC1 yielded only one product whose retention time and mass spectral pattern matched that of the standard of the NIH shift product, 3-chloro-2-hydroxybiphenyl (Table 3). Although the location of the hydroxyl group could not be precisely identified, the good match of this product with the NIH shift product's standard and a previous report of the NIH shift by this strain (Adriaens 1994) strongly suggest that the NIH shift was the only pathway followed by strain CSC1 with 2-chlorobiphenyl. The presence of the NIH shift was not directly observed with $M$. trichosporium OB3b, as the reported chlorohydroxybiphenyl products upon incubation with 2-chlorobiphenyl were not positively matched to the NIH shift standard (Lindner et al. 2000). The results here suggest a primary pathway followed by strain CSC1 with 2-chlorobiphenyl is epoxidation yielding the arene oxide intermediate and subsequent NIH shift of the ortho-substituted chlorine, whereas this cannot be concluded for M. trichosporium OB3b.

QSBR analysis of rates and relative influence of substituent electronics, sterics, and hydrophobicity

Both the slope of the initial rate-concentration points including the maximum rate, and the maximum normalized observed oxygen uptake rates were tested as dependent variables in QSBRs for both methanotrophs. As was reported in Lindner et al. (2003), the "linearized" rates (the slopes of the lines fit to the initial rates) resulted in the best correlations for $M$. trichosporium OB3b with small linearized rate values corresponding to large maximum normalized observed oxygen uptake rates. It is likely that the correlations with the maximum observed rates were not as robust, because no clear maximum oxygen uptake rate was observed for either 2methylbiphenyl or 2-hydroxybiphenyl. However, the overall trends as to which substituent property had greatest influence on rates were the same, regardless of whether linearized rates or maximum observed rates of M. trichosporium OB3b was used. As shown in Fig. 1 for biphenyl, 2-hydroxybiphenyl, and 2-chlorobiphenyl, all of the rate-substrate concentration curves obtained with strain $\mathrm{CSC} 1$ and the remainder of the substrates (not shown) yielded clear maxima. Unlike the QSBR models of $M$. trichosporium $\mathrm{OB} 3 \mathrm{~b}$, only the maximum rates of oxygen uptake observed with strain CSC1 yielded robust correlations. However, because the same trends in substituent effects on $M$. trichosporium OB3b oxidation activity were revealed regardless of the rate type used, comparison of the correlations between these two strains can be made.

\section{Linear correlations with strain CSC1 rates}

The QSBR analysis was performed to quantify the relative influence of electronic, steric, and "hydrophobic" nature of the ortho substituents on the oxidation rate. The only independent variable yielding a linear corre-
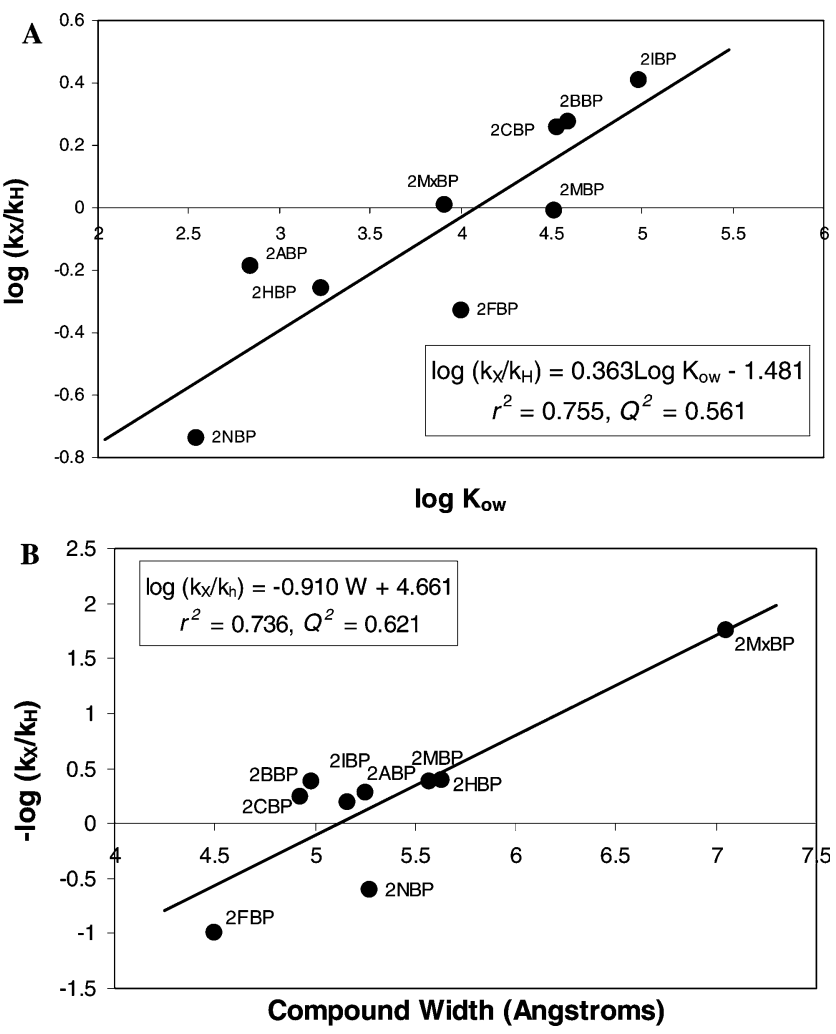

Fig. 2 Linear correlation between rates and descriptors. a Maximum oxygen uptake rates by strain CSC1 correlated with $\log K_{\text {ow }}$ (Eq. 1). b Slopes of the initial rate (including the maximum observed rate)-substrate concentration curves with $M$. trichosporium OB3b correlated with compound width $(W) .-\log \left(k_{x} / k_{H}\right)$ is used here because the slope and oxidation potential are inversely proportional (Lindner et al. 2003). Data used for $\mathbf{b}$ were obtained from Lindner et al. 2000

lation with strain CSC1 rates in conformity with the imposed selection criteria was $\log K_{\text {ow }}$ (Eq. 1), as plotted in Fig. 2a.

$$
\begin{aligned}
& \log \left(\frac{k_{\mathrm{X}}}{k_{\mathrm{H}}}\right)=0.363(0.185) \log K_{\mathrm{ow}}-1.481(0.737) \\
& \left(n=9, r^{2}=0.755, Q^{2}=0.561, S E=0.189, \mathrm{MSR}\right. \\
& \quad=0.036, F=21.567),
\end{aligned}
$$

where $k_{X}$ is the maximum oxygen uptake rate observed in the presence of the substituted biphenyl, $k_{H}$ is the maximum oxygen uptake rate observed in the presence of unsubstituted biphenyl, $r^{2}$ and $Q^{2}$ represent the correlation's goodness-of-fit to the observed data and predictive capability, respectively, MSR is the mean square regression of the residual, $F$ is $F$-ratio statistic. Numbers in parentheses represent the $95 \%$ confidence interval of the regression coefficient. The correlation in Eq. 1 implies that an increase in compound hydrophobicity results in an increase in oxidation activity by strain CSC1. This behavior has been observed previously by Parsons et al. (1987) in assessing biodegradation kinetics of Pseudomonas strain B1 with alkyl esters of para-aminobenzoic acid. These authors 
reasoned that the strong linear relationship obtained between the rates and compound lipophilicity is evidence that biodegradation rates of these compounds are determined by passive diffusion through the cell membrane. If whole-cell biodegradation processes are controlled by bonding to and transformation at the active site, electronic and/or steric effects show greater influence in correlations (Banerjee et al. 1984; Deardon and Nicholson 1987; Parsons et al. 1987). The strong linear correlation between oxygen uptake rates by strain CSC1 and $\log K_{\text {ow }}$ would thus suggest that the rate of uptake into and transport within the cell determines the overall rate of biodegradation by this strain.

Whereas none of the electronic descriptors resulted in linear correlations with $r^{2}$ values higher than 0.7 , it is interesting to note that the highest $r^{2}$ values were obtained with $\mathrm{C}_{9}: \mathrm{C}_{12}$, the sum of the charges on the carbons ortho, meta, and para to and including the substituted carbon $\left(r^{2}=0.611\right)$, and $\mathrm{C}_{8}: \mathrm{C}_{12}$, the sum of all of the charges on the carbons of the substituted ring $\left(r^{2}=0.605\right)$ (correlations not shown). Of the individual carbon charges $\left(\mathrm{C}_{8}-\mathrm{C}_{12}\right)$, the substituted carbon, $\mathrm{C}_{12}$, yielded a significantly higher $r^{2}$ value (0.489) compared to the other carbon charges (ranging from 0.006 to $0.173)$. This trend suggests the "preference" of this substituted carbon position during oxidation.

\section{Multilinear correlations with strain CSC1 rates}

Multivariate regression analysis further revealed the relative influence of electronic, steric, and hydrophobicity effects on the oxidation activity of strain $\mathrm{CSC}$. The strongest correlations between strain CSC1 rates and the electronic descriptors were obtained with $\log K_{\text {ow }}$, and no descriptor representing substituent size yielded acceptable multilinear correlations. Equations 2 and 3 show correlations with the highest $r^{2}$ values obtained in this analysis:

$$
\begin{aligned}
& \log \left(\frac{k_{\mathrm{X}}}{k_{\mathrm{H}}}\right)=-0.333(0.549) R+0.386(0.181) \log K_{\mathrm{ow}} \\
&-1.684(0.781) \\
&\left(n=9, r^{2}=\right. 0.821, Q^{2}=0.611, S E=0.175, \\
& \mathrm{MSR}=0.031, F=13.720), \\
& \log \left(\frac{k_{\mathrm{X}}}{k_{\mathrm{H}}}\right)=-1.665(3.093) \mathrm{C}_{8}: \mathrm{C}_{12} \\
& \quad+0.266(0.256) \log K_{\mathrm{ow}}-1.441(0.729) \\
&\left(n=9, r^{2}=0.810, Q^{2}=0.532, S E=0.159,\right. \\
&\mathrm{MSR}=0.032, F=12.783) .
\end{aligned}
$$

Figure $3 \mathrm{a}$ and $\mathrm{b}$ shows the $\log \left(k_{x} / k_{H}\right)$ values predicted by Eqs. 2 and 3 plotted against observed values. No acceptable correlations resulted with Hammett constants or the field effects parameter $F$. In all cases,

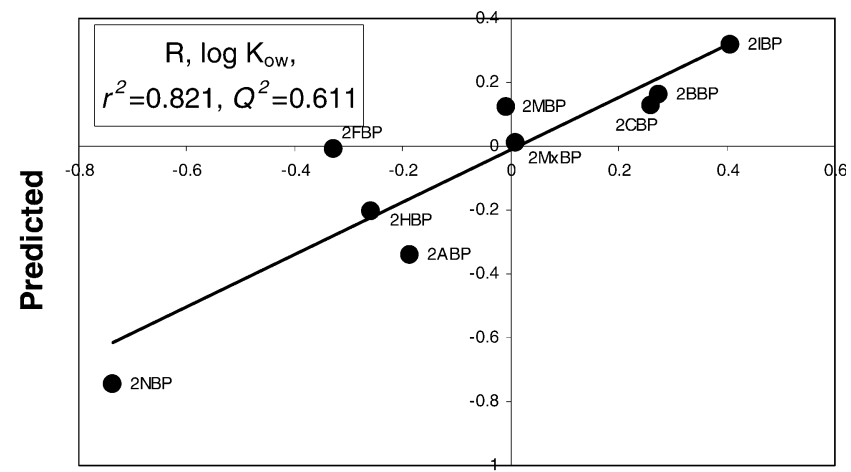

Observed

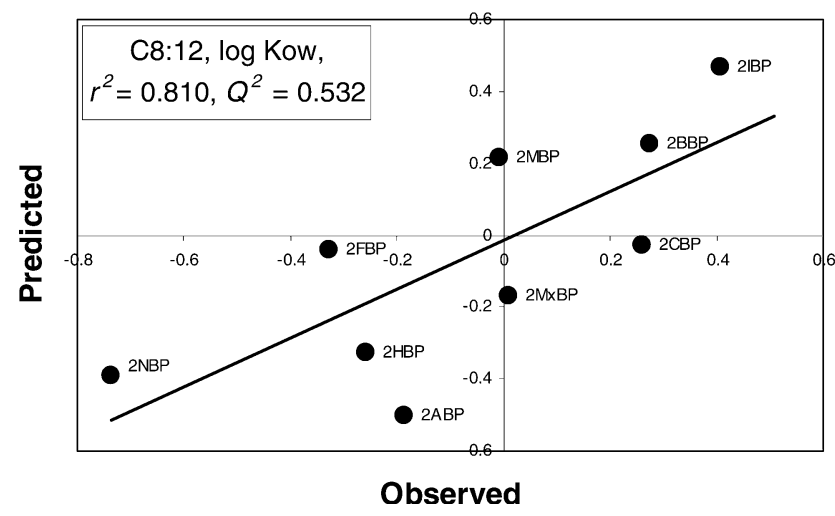

Fig. 3 Comparison of observed and predicted values of oxygen uptake rates for selected models correlating the maximum observed oxygen uptake rates and substituent descriptors (a Eq. 2; b Eq. 3). See Table 1 for abbreviations of substrates

$\log K_{\text {ow }}$ resulted in higher $r^{2}$ values than the hydrophobicity parameter $\pi$.

Despite the strong influence of compound hydrophobicity as discussed earlier, Eqs. 2 and 3 show that electronics also play a role in influencing oxidation activity of strain CSC1. A decrease in resonance parameter $R$ and in $\mathrm{C}_{8}: \mathrm{C}_{12}$ (thus a greater electron density on the substituted ring) and an increase in compound hydrophobicity increase oxidation activity of strain CSC1. The findings that greater mesomeric effects of the substituent and higher electron density on the substituted ring promote greater oxidation activity are consistent with the electron-deficient nature of the active site of sMMO (Shu et al. 1997).

Of the correlations between strain CSC1 rates and the charges of individual carbons on the substituted ring $\left(\mathrm{C}_{8}-\mathrm{C}_{12}\right)$ and $\log K_{\mathrm{ow}}$, the one yielding the highest $r^{2}$ included the charge of the substituted carbon $\left(\mathrm{C}_{12}\right)$ (correlation not shown; $r^{2}=0.784, \quad \mathrm{SE}=0.192$, MSR $=0.037, F=10.883$ ). As previously observed with the linear correlations, this suggests the importance of this location during oxidation of the biphenyl substrates. Recall that the NIH shift product, formed only by an initial attack at the region of the substituted carbon, was the only product observed in the 2-chlorobiphenyl incubation studies (Table 3). The importance of this 
region during oxidation of these compounds by strain CSC1 is further supported by a strong, positive correlation between oxidative activity and the difference of the charges of the substituted aromatic carbon and the aromatic carbon-bonded substituent $\left(\delta_{C-\mathrm{X}}\right)$ and $\log K_{\mathrm{ow}}$ (correlation not shown; $r^{2}=0.799, \quad \mathrm{SE}=0.185$, $\mathrm{MSR}=0.034, F=11.937)$.

\section{QSBR comparison with $M$. trichosporium OB3b}

As previously described, the linearized rates of oxygen uptake by $M$. trichosporium OB3b resulted in superior correlations with greater predictive capability than the maximum normalized oxygen uptake rates; however, a decrease in the linearized rates directly corresponded to an increase in maximum observed oxygen uptake rates. The linearized rates by $M$. trichosporium OB3b showed strong positive linear correlation with compound width $(W)$ in a correlation with an $r^{2}$ value of 0.734 and $Q^{2}$ value of 0.621 (Lindner et al. 2003), as shown in Fig. 2 b. The influence of compound size on oxidation activity of $M$. trichosporium OB3b was further observed in multilinear correlations, with the most robust multilinear correlation resulting with descriptors of the sum of the carbon charges on the substituted ring $\left(\mathrm{SUMC}_{8}: \mathrm{C}_{12}\right)$ and $W$ accompanied by an $r^{2}$ of 0.828 and $Q^{2}$ of 0.665 (Lindner et al. 2003). As for the strain CSC1 QSBR results, the individual carbon on the substituted ring showing the strongest influence on rates of oxidation by $M$. trichosporium $\mathrm{OB} 3 \mathrm{~b}$ was the substituted carbon $\left(\mathrm{C}_{12}\right)$, and the resulting correlation with compound width $(W)$ possessed an $r^{2}$ of 0.819 and $Q^{2}$ of 0.673 . Also, the charge difference across the substituted carbon-substituent bond correlated very strongly with oxidation rates.

As shown with strain CSC1, oxidation activity of $M$. trichosporium $\mathrm{OB} 3 \mathrm{~b}$ was increased with increasing electron density on the substituted ring and with increasing charge difference along the substituted carbon-substituent bond. Thus, both strains, expressing sMMO, show preference for electron-rich substrates, consistent with the electron-deficient nature of active site of sMMO, and the significant influence of the site of the substituted carbon is consistent with its importance during oxidation (Lindner et al. 2003).

Unlike strain CSC1, however, compound hydrophobicity showed no influence on rates observed with $M$. trichosporium OB3b. The oxidation activity of $M$. trichsporium $\mathrm{OB} 3 \mathrm{~b}$ increases with increasing compound size, suggesting a "buttressing effect," where the reaction at the active site is benefited by larger compounds that are more effectively held in position by the enzyme (Hansch and Caldwell 1991). This result is consistent with the strong preference by $M$. trichosporium $\mathrm{OB} 3 \mathrm{~b}$ for the para position during oxidation, thus implying an inability of the biphenyl molecule to rotate freely in the active site. Furthermore, the dependence of rates of $M$. trichosporium OB3b oxidation of ortho-substituted biphenyls on both compound electronics and size supports the hypothesis that the rate-determining step involves the active site and not the cell membrane (Parsons et al. 1987).

\section{Conclusions}

By providing evidence of oxidation of various orthosubstituted biphenyls, this study reports an even broader range of substrate specificity of whole cells of strain CSC1 than previously reported. The NIH-shift pathway may be preferred by this organism as observed by the NIH-shift product of 2-chlorobiphenyl being the only product formed. Whereas different oxidation rates by the methanotrophs were observed with the biphenyls, QSBR analysis enabled determination of trends in rate differences between the two strains. The QSBRs obtained from the maximum oxygen uptake rates observed in the presence of the nine substrates suggest the significance of both electron density of the substituted ring and compound hydrophobicity in determining oxidation activity of strain $\mathrm{CSC} 1$, with the latter characteristic showing high relevance to the correlations obtained. $M$. trichosporium OB3b also shows a similar preference for compounds with greater electron density on the substituted ring; however, compound size, and not hydrophobicity, clearly plays an influential role in influencing oxidation rates. What is suggested by these results is that the rate-determining step involved in strain CSC1 oxidation is transport-related, either through the cell membrane or to the active site, whereas the rate of oxidation of these compounds by $M$. trichosporium OB3b is determined by reaction and/or release at the active site. Studies at the enzyme level will verify determination of whether these physiological differences in the two strains attribute to their observed differences in oxidation behavior and will provide a first basis for understanding differences in and enabling prediction of oxidation behavior of methanotrophs under sMMO expression.

Acknowledgements We would like to thank Ms. Adriana Pacheco, a graduate research assistant in the Department of Environmental Engineering Sciences at the University of Florida, for her assistance in the laboratory. We wish to thank Dr. Ning Chen of ChemTrace in Fremont, Calif., for performing the Gaussian ab initio analysis. Fellowships to Angela S. Lindner from the NIH Cellular Biotechnology Training Program (grant 2T32GM08353-06) and the American Association of University Women are gratefully acknowledged.

\section{References}

Adriaens P (1994) Evidence for chlorine migration during oxidation of 2-chlorobiphenyl by a Type II methanotroph. Appl Environ Microbiol 60:1658-1662

Adriaens P, Grbić-Galić D (1994) Cometabolic transformation of mono- and dichlorobiphenyls and chlorohydroxybiphenyls by methanotrophic groundwater isolates. Environ Sci Technol 28:1325-1330 
Banerjee S, Howard PH, Rosenberg AM, Dombrowski AE, Sikka H, Tullis DL (1984) Development of a general kinetic model for biodegradation and its application to chlorophenols and related compounds. Environ Sci Technol $18: 416-422$

Bowman J (2000) The methanotrophs: the families Methylococcaceae and Methylocystaceae. In: Dworkin M, et al (eds) The prokaryotes: an evolving electronic resource for the microbiological community, 3rd edn. Springer, Berlin Heidelberg New York, release 3.1., http://link.springerny.com/link/service/ books/10125/.

Bowman JP, Sayler GS (1994) Maximization of Methylosinus trichosporium OB3b soluble methane monooxygenase production in batch culture. Biodegradation 5:1-11

Bowman JP, Sly LI, Nichols PD, Hayward AC (1993) Revised taxonomy of the methanotrophs: description of Methylobacter gen. nov., emendation of Methylococcus, validation of Methylosinus and Methylocystis species and a proposal that the family Methylococcaceae includes only the group I methanotrophs. Int J Syst Bacteriol 43:735-753

Brigmon R (2002) Methanotrophic bacteria: use in bioremediation. In: Bitton G (ed) Encyclopedia of environmental microbiology. Wiley, New York, pp 1936-1944

Brusseau GA, Tsien H-C, Hanson RS, Wackett LP (1990) Optimization of trichloroethylene oxidation by methanotrophs and the use of a colorimetric assay to detect soluble methane monooxygenase. Biodegradation 1:19-29

Burrows KKJ, Cornish A, Scott D, Higgins IJ (1984) Substrates specificities of the soluble and particulate methane mono-oxygenases of Methylosinus trichosporium OB3b. J Gen Microbiol 130:3327-3333

Choi D-W, Kunz RC, Boyd ES, Semrau JD, Antholine WA, Han J-I, Zahn JA, Boyd JM, del la Mora AM, DiSpirito AA (2003) The membrane-associated methane monooxygenase (pMMO) and pMMO-NADH:quinone oxidoreductase from Methylococcus capsulatus Bath. J Bacteriol 185:5755-5764

Conrad R (1996) Soil microorganisms as controllers of atmospheric trace gases $(\mathrm{H} 2, \mathrm{CO}, \mathrm{CH} 4, \mathrm{OCS}, \mathrm{N} 2 \mathrm{O}$, and $\mathrm{NO})$. Microbiol Rev 60:609-640

Dalton H, Golding BT, Waters BW, Higgins R, Taylor JA (1981) Oxidations of cyclopropane, methylcyclopropane, and arenes with the mono-oxygenase system from Methylococcus capsulatus. J Chem Soc Chem Commun 10:482-483

Dalton H, Wilkins PC, Jiang Y (1993) Structure and mechanism of action of the hydroxylase of soluble methane monooxygenase. In: Murrell JC, Kelly DP (eds) Microbial growth on $\mathrm{C}_{1}$ compounds. Intercept, Andover, pp 65-80

Damborsky J, Manova K, Kuty M (1996) A mechanistic approach to deriving quantitative structure biodegradability relationships. In: Peijnenburg WJGM, Damborsky J (eds) Biodegradability prediction. Kluwer, Dordrecht, pp 75-92

Dearden JC, Nicholson RM (1987) Correlation of biodegradability with atomic charge difference and superdelocalizability. In: Kaiser KLE (ed) QSAR in environmental toxicology-II. D Reidel, Dordrecht, pp 83-89

DiSpirito A, Gulledge J, Shiemke AK, Murrell JC, Lidstrom ME, Krema CL (1992) Trichloroethylene oxidation by the membrane-associated methane monooxygenase in type I, type II, and type X methanotrophs. Biodegradation 2:151-164

Eriksson L, Johansson E, Wold S (1997) Quantitative structure-activity relationship model validation. In: Chen F, Schuurmann G (eds) Quantitative structure-activity relationships in environmental sciences-VII. Setac, Pensacola, pp 381-397

Eriksson L, Jaworska J, Worth AP, Cronin MTD, McDowell RM, Gramatica P (2003) Methods for reliability and uncertainty assessment and for applicability evaluations of classificationand regression-based QSARs. Environ Health Perspect 111:1361-1375

Foresman JB, Frisch A (1996) Exploring chemistry with electronic structure methods, 2nd edn. Gaussian, Pittsburgh

Gaussian 94 software (revision A.1) (1996) Gaussian, Pittsburgh
Green J, Dalton H (1989) Substrate specificity of soluble methane monooxygenase-mechanistic implications. J Biol Chem 264:17698-17708

Grosse S, Laramee L, Wendlandt K-D, McDonald IR, Miguez CB, Kleber H-P (1999) Purification and characterization of the soluble methane monooxygenase of the type II methanotrophic bacterium. Methylocystis sp. strain WI 14. Appl Environ Microbiol 65:3929-3935

Hansch C, Caldwell J (1991) The structure-activity relationship of inhibitors of serotonin uptake and receptor binding. J Comput Aided Mol Des 5:441-453

Hansch C, Leo A, Hoekman D (1995) Exploring QSAR: hydrophobic, electronic, and steric constants, ACS Professional Reference Book. American Chemical Society, Washington

Henry SM, Grbić-Galić D (1990) Effect of mineral media on trichloroethylene oxidation by aquifer methanotrophs. Microb Ecol 20:151-169

Hermens J, Balaz S, Damborsky J, Karcher W, Muller M, Peijnenburg W, Sabljic A, Sjostrom M (1995) Assessment of QSARs for predicting fate and effects of chemicals in the environment: an international European project. SAR QSAR Environ Res 3:223-236

Hršak D (1996) Cometabolic transformation of linear alkylbenzenesulphonates by methanotrophs. Water Res 30:3092-3098

Jezequel SG, Higgins IJ (1983) Mechanistic aspects of biotransformations by the monooxygenase systems of Methylosinus trichosporium OB3b. J Chem Tech Biotechnol 33B:139144

Kitson FG, Larsen BS, McEwen CN (1996) Gas chromatography and mass spectrometry: a practical guide. Academic, San Diego

Kohler H-PE, Kohler-Staub D, Focht DD (1988) Degradation of 2-hydroxybiphenyl and 2,2'-dihydroxybiphenyl by Pseudomonas sp. strain HBP1. Appl Environ Microbiol 54:2683-2688

Kohler H-PE, van der Maarel MJEC, Kohler-Staub D (1993) Selection of Pseudomonas sp. strain HBP1 Prp for metabolism of 2-propylphenol and elucidation of the degradative pathway. Appl Environ Microbiol 59:860-866

Lindner AS, Adriaens P, Semrau JD (2000) Transformation of ortho-substituted biphenyls by Methylosinus trichosporium OB3b: substituent effects on oxidation kinetics and product formation. Arch Microbiol 174:35-41

Lindner AS, Whitfield C, Chen N, Semrau JD, Adriaens P (2003) Quantitative structure-biodegradation relationships for orthosubstituted biphenyl compounds oxidized by Methylosinus trichosporium OB3b. Environ Toxicol Chem 22:2251-2257

Lontoh S, DiSpirito AA, Semrau JD (1999) Dichloromethane and trichloroethylene inhibition of methane oxidation by the membrane-associated methane monooxygenase of Methylosinus trichosporium OB3b. Arch Microbiol 171:301-308

Montgomery DC, Peck EA (1982) Introduction to linear regression analysis. Wiley, New York

Parsons JR, Opperhuizen A, Hutzinger O (1987) Influence of membrane permeation on biodegradation kinetics of hydrophobic compounds. Chemosphere 16:1361-1370

Prior SD, Dalton H (1985) The effect of copper ions on membrane content and methane monooxygenase activity in methanolgrown cells of Methylococcus capsulatus (Bath). J Gen Microbiol 131:155-163

Reeburgh WS (1996) Soft spots in the global methane budget. In: Lidstrom ME, Tabita FR (eds) Microbial growth on C1 compounds. Kluwer, Dordrecht, pp 334-342

Shu L, Nesheim JC, Kauffmann K, Munck E, Lipscomb JD, Que L Jr (1997) An $\mathrm{Fe}_{2}(\mathrm{IV}) \mathrm{O}_{2}$ diamond core structure for the key intermediate Q of methane monooxygenase. Science 275:515518

Stanley SH, Prior SD, Leak DJ, Dalton H (1983) Copper stress underlies the fundamental change in intracellular location of methane mono-oxygenase in methane-oxidizing organisms: studies in batch and continuous cultures. Biotech Lett 5:487492

Taylor JK (1990) Statistical techniques for data analysis. Lewis, Boca Raton 
Topliss JG, Edwards RP (1979) Chance factors in studies of quantitative structure-activity relationships. J Med Chem 22:1238-1244

Uchiyama H, Nakajima T, Yagi O, Tabuchi T (1989) Aerobic degradation of trichloroethylene by a new type II methaneutilizing bacterium, strain M. Agric Biol Chem 53:2903-2907
Wilkins PC, Dalton H, Samuel CJ, Green J (1994) Further evidence for multiple pathways in soluble methane-monooxygenase-catalyzed oxidation from the measurement of deuterium kinetic isotope effects. Eur J Biochem 226:555-560 